\title{
EIN SCHLUSSWORT \\ ZU CEDERSCHIÖLDS AUSGABE DER BEVIS SAGA.
}

Zu Cederschiölds aufsatz 'Ueber die ausgabe der Bevis saga' Beitr. $23,257 \mathrm{ff}$. gestatte ich mir folgende erwiderung, indem ich dabei gleich bemerke, dass ich mich bemühen werde, einen weniger hochfahrenden ton anzuschlagen als C.; ausdrücke wie 'lächerlich' und 'unwissenheit' werden in meinen darlegungen z.b. nicht vorkommen.

Recapitulieren wir zunächst kurz die sachlage. C. legt seiner ausgabe der Bevis saga den cod. Holm. membr. 6, $4^{0}$ (B) zu grunde, soweit er vollständig, und ergänzt die lücken durch cod. Holm. 7 fol. (C) bez. durch zwei demselben nahe stehende papierhss. $(\gamma \delta)$. Bezüglich der verwertung dieser letzteren hss. für die teile der saga, welche in beiden fassungen vorliegen, erklärt er s. LxIv seiner FSS., er habe aus ihnen 'alle von unserem texte abweichenden lesarten aufgenommen, so dass nur die so gut wie wertlosen abweichungen nicht angemerkt worden' seien. Diese äusserung liegt gedruckt vor; der zusammenhang, in dem sie vorgebracht wird, ändert an ihrem sinne nicht das mindeste (vgl. C. s. 262). Wenn er (abgesehen von einer späteren stelle der einleitung s. ccxL) jetzt (s. 262) diese in denkbar klarster form abgegebene zusage in merkwürdig verclausulierter weise dahin abgeschwächt, er habe 'eine hauptsächlich vom nordisch-philologischen standṕpunkte aus einigermassęn vollständige sammlung der abweichenden lesarten ... zu geben versucht', so muss ihm die, wie mir scheint nicht ganz leicht $\mathrm{zu}$ tragende verantwortung dafür zugeschoben werden. 
Er hat ferner weder die franz. hss., deren eine ihm aus meinen Beiträgen (1876) s. 136 bekannt sein musste,1) benutzt, noch auch die englische, 1838 erschienene, oder die gälische fassung, mit englischer übersetzung gedruckt 1880, zur vergleichung herangezogen. Und hätte er wenigstens noch seine zusage erfüllt, alle inhaltlichen ('sachlich' verstehe ich in meinem aufsatze ebenso wie Elis s. s. xxxvir im gegensatz zu 'graphisch', wodurch C.'s darauf bezügliche auslassungen s. 265 ff. gegenstandslos werden) varianten von $\mathrm{C} \gamma \delta$ zu notieren, so träfe ihn zwar immer noch der vorwurf, eine minderwertige hs. zu grunde gelegt zu haben, aber er hätte doch dankenswertes, vollständiges material geliefert; dass das nicht geschehen, lehrt ein blick auf meine nachträge. Ja nicht einmal die recht bescheidene aussicht, eine 'hauptsächlich vom nordischphilologischen standpunkte aus einigermassen vollständige sammlung der abweichenden lesarten' zu erhalten, hat C. verwirklicht; an einer ganzen anzahl von stellen, wo die lesung von B sich auch ohne die hinzunahme fremder redactionen als mangelhaft erwies, hat der herausgeber die wichtigen varianten der anderen hss. anzuführen unterlassen; vgl. meine note $\mathrm{zu}$ s. 216 z. 25, aus der er sehen kann, dass die frage, ob Bevis von elf oder zwölf rittern angegriffen wird, doch nicht so bedeutungslos ist wie er glaubt (s. 280); ferner meine anmerkungen zu s. 216, 38 (vgl. auch Beitr. s. 42 no. 40). 232, 6 (Beitr. s. 46 no. 118). 248, 34 f. 251, 15 f. 57. 251, 16. 253, 33 f. 46 f. 256,50 f. 265,40 f.

Alle diese punkte stehen fest, und weder die früheren noch etwaige zukünftige argumentationen C.'s werden im stande sein, sie $\mathrm{zu}$ beseitigen.

C. behauptet, ich hätte ihm unrecht getan durch die verschweigung des umstandes, dass er von jeder saga nur éine redaction mitzuteilen beabsichtigt habe. Nun, ich habe keineswegs 'ausser betracht gelassen, dass auch von romantischen sqgur verschiedene redactionen existieren können' (s. 261); ich verstehe darunter aber nur solche fälle, wo, um mich etwas

1) Wenu Firnnin Didot mir, dem Dentachen, 1876, also wenige juhre pach dem krjege, ausilricklich die erlanhnis verweigerte, eine copie vou der hs. zu nehmen, so ist das wol erklïrlich; ein Schwede hiitte grwis cincu besseren erfolg erzielt. 
ausserlich auszudriicken, die differenzen so stark sind, dass es Immïglich wird, diesselben in variantenform daszustellen, wie das \%.b. bei lı. I) der IAlis saga im verhältnis zum Upsalaer colex und bei cod. IIolm. 6, 4" der Jjalar .Jóns saga, verglichen mit. Jiroarsons text, der fall ist. Jie verschiedenen mss. der Bevis saga repräsentieren dagegen nur zwei hss.-klassen; das besagt schon die von C. s. 265 ausgehobene bemerkung in meinem ersten aufsatze. Und dazu kommt die früher besprochene erklärung des herausgebers selbst, dass er in diesem falle anders verfahren wolle. Ich fühle mich also von dem vorwurf durchaus frei, in tendenziöser absicht mich einer verschweigung schuldig gemacht zu haben. Im übrigen aber haben wir es gerade hier mit einem grundirrtum C.'s zu tun. Es ist nicht richtig, dass jeder herausgeber, wie er vorauszusetzen scheint, das recht hat, sich selbst eine eigene kritische methode $\mathrm{zu}$ construieren und dann zu verlangen, dass seine leistung bloss von diesem standpunkte aus, als einem gegebenen, beurteilt werde. Ein text der nur auf einen eng beschränkten kreis von interessenten zu rechnen hat, muss gleich das erste mal in einer form geboten werden, die für alle weitere arbeit eine feste grundlage liefert - eine forderung deren berechtigung gewis alle einsichtigen anerkennen werden. Ist das nicht der fall, so halst der herausgeber dem benutzer weitere arbeit auf und erschwert zugleich buchhändlerisch die veröffentlichung einer vollständigeren ausgabe. C. erkennt dies princip nicht an: 'das ganze material zu bieten, das möglicherweise zur vergleichung mit den franz. texten nötig werden könnte, hatte ich weder beabsichtigt noch versprochen', bemerkt er (s. $263^{1}$ )) und gibt damit das selbst zu, was ich hauptsächlich hatte nachweisen wollen.

Eine ganz andere frage ist die nach der aufnahme 'formeller' varianten, wie sie C. nennt; hier können die meinungen in der tat auseinander gehen, wie ich das vor erscheinen von C.'s artikel selbst (Publ. of the Mod. Lang. Assoc. of America, vol. 13, Baltimore 1898, s. 554) offen ausgesprochen habe. Indessen hat kein recensent meiner Elis saga, auch C. nicht, mix einen vorwurf daraus gemacht, dass ich in dieser ausgabe selbst in der anführung dieser gruppe von varianten vollständigkeit angestrebt habe. 
C. meint ferner, das factum, dass prof. Stimming mir seine copien der zwei hss. des ältesten franz. textes geliehen habe, hätte stärker betont werden sollen als ich es getan; er glaubt nicht fehlzugreifen, wenn er gerade in dem entleihen dieser copien den eigentlichen entstehungsgrund von meiner strengen kritik seiner ausgabe erblicke. 'Und ich kann nicht umhin, seine art, sich über meine ausgabe zu äussern, mit der übermütigen kritik zu vergleichen, die ein schüler mit hilfe des in seine hände gelangten schlüssels des lehrers an der von einem mitschüler ohne dieses unschätzbare hilfsmittel angefertigten ïbersetzung übt' (s. 259). C. irrt sich. Mir standen die Pariser hss. in derselben weise zur verfügung wie Stimming; hätte dieser die abschrift nicht schon genommen und die liebenswürdigkeit gehabt sie mir zu leihen, so würde ich, ehe ich an die herausgabe des Sir Beves und an die nähere betrachtung der saga gieng, die nochmalige ${ }^{1}$ ) reise nach Paris für diesen zweck ebensowenig gescheut haben, wie ich versäumt habe, vor abfassung meiner abhandlung über die Elis saga den damals noch nicht gedruckten Elie de St. Gille derselben bibliothek zu studieren, oder zum zweck der herstellung eines kritischen textes der me. Ipomandonromanze von den beiden afranz. hss. der - beiläufig ca. 10,000 verse langen - quelle im Brit. museum copien zu nehmen. Beides erachtete ich für ganz selbstverständlich, und so erschien es mir auch als ziemlich irrelevant für die sache, wo ich mein unentbehrliches kritisches material im vorliegenden falle herbekommen hatte; nur die schuldige danksagung gab zur andeutung dieser verhältnisse veranlassung. Dass übrigens der für mich nicht. sonderlich schmeichelhafte vergleich mit dem 'schlüssel' herzlich schlecht passt, bedarf keiner ausführung.

Ich habe C. immer für einen selır sorgsamen und gewissenhaften handschriftenleser gehalten, ${ }^{2}$ ) und darin macht mich

1) Vgl. oben s. 415 anm 1.

2) Dieser selben meinung hatte ich Beitr. 19, 64') folgenden ansilurk geliehen: 'C.'s textabdriicke werlen im allgemeinen mit recht. wrgen ihrer ausserordentlichen akribie gerühmt, Ich weiss nicht, wic 4 dazu kommt. darin etwas wie spott, zn wittem (s. 276): ich habe nichts dergleichen locabsichtigt, und muss mich gegen cine solehe willkibliche miterstellung verwaliren. 
aurh der unstand nichi, irre, dass sein abdruck einer kurzen handschriftlichen noti\% aus dem 17. jh. vier lesefehler enthält (vol. Publ. a. a. o. s. 5.44) und er in den varianten zur Clarus saga zweimal ohne erklärung den casus abgeändert hat (vgl. (las. s. $\left.557^{2}\right)$ ). Sein urteil ïber mich ist freilich weniger fleundlich: er spricht meinem ergänzenden variantenapparate zuverlässigkeit und genauigkeit ab (s.276). Die sache liegt so. Als ich für die An. sagabibliothek die herausgabe der Flóres saga iibernommen und zugleich beschlossen hatte, der Bevis saga ein eingehenderes studium zuzuwenden, nahm ich im herbst 1892 zunächst einen kurzen aufenthalt in Kopenhagen, um über das handschriftliche material einen vorläufigen iiberblick zu gewinnen. Bei dieser gelegenheit collationierte ich u. a. unter ungünstigen beleuchtungsverhältnissen die fragmente $A$ und $D$ der Bevis saga, und hielt dann bei ausarbeitung des apparates meine collation derselben für genügend, zumal C. gerade von A besonders reichliche varianten mitgeteilt hatte; doch stiegen mir schon bald nach dem druck des aufsatzes bedenken auf, ob ich darin recht getan hätte. Die hss. C $\gamma \delta$, also das hauptsächlichste material für meine arbeit, dagegen habe ich lange zeit hier benutzen dürfen und dabei namentlich gelegenheit gehabt, der oft recht schwer lesbaren hs. C das eingehendste studium zu widmen; ich war in der lage, die correctur in aller musse mit den mss. zur seite zu lesen, und ich bin mir bewusst, den apparat mit der denkbar grössten gewissenhaftigkeit zusammengestellt zu haben. Höchstens könnte - um ja nichts zu verschweigen - das misgeschick, dass zu einer zeit, wo die eine der papierhss. bereits wider weggeschickt war, auf dem wege von Leipzig nach Halle ein revisionsbogen durch die post in verlust geraten ist, vielleicht ein paar kleine ungenauigkeiten verschuldet haben. Die hss. sind allgemein zugänglich: jeder interessent, vor allem C. selbst, kann sich mit leichtigkeit überzeugen, ob ich zu viel behaupte. Ja vielleicht ist eine erneute einsicht in die hss. zur beurteilung der sachlage nicht einmal nötig; fast sämmtliche correcturen meines apparates durch C. (s. 277 f.) beziehen sich auf die fragmente $\mathrm{D}$ und $\mathrm{A}$; nur zwei gesicherte berühren $\gamma \delta$, und zwar handelt es sich das erste mal s. 209,4 um ein druckversehen, indem vor 'unnit ok $\gamma \delta$ ' add. ausgefallen 
ist: es war von vornherein unwahrscheinlich, dass beide hss. hann hafoi undir unnit olk lag | lesen sollten; unmittelbar darauf liegt eine unterlassungssünde vor, indem das fehlen von riddari in $\gamma \delta$ nicht angemerkt worden ist. C. hat etwa ein zehntel meiner liste verglichen: wenn, wie ich zu hoffen wage, das verhältnis für die übrigen neun zehntel der saga dasselbe ist, dass also in den ca. 3000 einzelangaben sich, was die haupthss. anlangt, nur etwa zehn druckversehen und zehn auslassungen sollten entdecken lassen, so darf ich wol behaupten, dass sich schwerlich jemand finden wird, der eine solche minutiöse arbeit genauer macht. C. müsste diese sachlage ja wol eigentlich auch bemerkt haben; sein überaus schroffes urteil über meine collation, das er dann scrupellos auf alle meine bisherigen publicationen ausdehnt (s. 276), scheint eine solche voraussetzung jedoch völlig auszuschliessen.

Dass ich A und D nicht zur nochmaligen einsicht für die correctur hierher erbeten habe, wodurch auch diese versehen vermieden worden wären, bedaure ich jetzt selbst sehr.

Die zwölf zeilen lexicalischer bemerkungen, über welche C. sich behaglich auf fast drei seiten verbreitet, gebe ich ihm gern preis, und bemerke nur, dass den ausdruck $\alpha_{\alpha} \pi \alpha \xi \lambda \varepsilon \gamma \delta \dot{\mu} \mu \varepsilon \nu o \nu$ ausser ihm schwerlich jemand absolut auffassen wird; er meint natürlich: nach ausweis unserer bisherigen, natürlich nicht vollständigen wörterbücher.

So viel für diesmal. Auf andere punkte werde ich gelegenheit haben, in meiner kritischen ausgabe der Bevis saga zurückzukommen, die ich erst dann zu veröffentlichen gedenke, wenn Stimmings lang ersehnter text des ältesten Beuve erschienen und damit für diesen sagenstoff ein neues, unmittelbares interesse wachgerufen sein wird.

Nur noch eine bemerkung zum schluss. ( möchte gern (s. 260) einen widerspruch construieren zwischen meines recension der FSS. in der Literaturzeitung 1885 und dem in meinem aufsatz von 1894 njedergelegten urteil. Auch darin ist er im unrecht. Wenn meine ansicht über seine Bevis saga jetzt, wo ich die hss. selbst eingesehen habe, ungünstiger geworden ist, so kann ihn das nach meinen ansfïlırmgen kaum wundern. Im übrigen aber halte ich sein buch anch jetat noch fiir wort. voll, wenn auch nicht als absuhliessende leistung, so doch als 
solır wichtigo materialiensammlung; ihr habe ich in meinen ammerkungen zur frlires saga und zur Ívens saga viele interasiante parallelstellen entmommen und forner in meinen notizen zur Komrals saga (Publ. a. a. o. s. 547 ff.) das von C. gebotene mit gutem willen llach einer seite hin zu ergänzen versucht -. wol die wirksamste art, wie man seine achtung vor der literarischen leistung eines anderen bekunden kann.

BRESLAU, nov. 1898.

E. KÖLBING.

\section{ERWIDERUNG.}

Durch die güte der redaction bin ich im stande, gleichzeitig mit dem erscheinen des obigen 'Schlusswortes' von prof. Kölbing meinerseits auf den erneuten angriff zu erwidern. Ich werde mich jedoch, um die güte der redaction und die geduld des lesers nicht zu misbrauchen, sehr kurz fassen.

Betreffend den von $K$. gerügten ton meines aufsatzes ('Ueber die ausgabe der Bevers saga') mögen wol andere umbefangener urteilen, ob dieser ton schärfer war, als die art des von $K$. (in seinen 'Studien zur Bevis saga') gegen mich gerichteten angriffs es berechtigte.

Das 'Schlusswort' K.'s besteht hauptsächlich in der widerholung einiger der beschuldigungen, die er bereits in seinen Studien gegen mich gerichtet hatte. Da ich auf diese beschuldigungen schon hinlänglich in meinem früheren aufsatz ('Ueber die ausgabe' etc.) geantwortet zu haben glaube, genügt es mir jetzt auf diesen aufsatz hinzuweisen. Uebrigens wird der kundige leser wahrscheinlich ohne fingerzeige finden, dass $\mathbf{K}$. im Schlussworte nichts neues zur hauptfrage - über die begrenzung des variantenapparats - beigesteuert hat, und dass er keinen ernstlichen versuch gemacht hat, zwischen entbehrlichen und unentbehrlichen varianten eine sicherere grenze zu ziehen.

Wesentlich neu ist aber die behauptung: 'an einer ganzen 